\title{
Hierarchy of equations for the energy functional of the density-functional theory
}

\author{
Á. Nagy \\ Institute of Theoretical Physics, Kossuth Lajos University, H-4010 Debrecen, Hungary
}

(Received 11 June 1992; revised manuscript received 23 November 1992)

\begin{abstract}
A hierarchy of equations has been derived for the energy functionals of the density-functional theory using the virial theorem and the Levy-Perdew relation. In the local-density approximation, the solution of the equations of hierarchy for the kinetic and exchange energies provides the well-known ThomasFermi expression for the kinetic energy and the Slater-Gáspár-Kohn-Sham expression for the exchange. The truncation of the hierarchies of the kinetic and exchange energies results in rigorous lower bounds to the kinetic energy and upper bounds to the exchange energy in the plane-wave approximation.
\end{abstract}

PACS number(s): $31.20 .5 y, 71.10 .+x, 05.30 . F k$

\section{INTRODUCTION}

Recently Kugler [1] has derived an exact hierarchy of coupled equations for the kinetic-energy functional of an inhomogeneous system of noninteracting fermions. The purpose of this paper is to obtain a hierarchy of equations for the energy functions of the exact density-functional theory.

The main results of this paper are as follows: the first,

$$
\begin{aligned}
F^{\prime}(\mathbf{r})+T^{\prime}(\mathbf{r})= & -\mathbf{r} \cdot \nabla F^{\prime}(\mathbf{r}) \\
& -\int d \mathbf{r}_{1} n\left(\mathbf{r}_{1}\right) \mathbf{r}_{1} \cdot \nabla_{1} F^{\prime \prime}\left(\mathbf{r}, \mathbf{r}_{1}\right) d \mathbf{r}_{1},
\end{aligned}
$$

the second,

$$
\begin{aligned}
F^{\prime \prime}\left(\mathbf{r}, \mathbf{r}_{1}\right)+T^{\prime \prime}\left(\mathbf{r}, \mathbf{r}_{1}\right)= & -\left(\mathbf{r} \cdot \boldsymbol{\nabla}+\mathbf{r}_{1} \cdot \nabla_{1}\right) F^{\prime \prime}\left(\mathbf{r}, \mathbf{r}_{1}\right) \\
& -\int d \mathbf{r}_{2} n\left(\mathbf{r}_{2}\right) \mathbf{r}_{2} \cdot \nabla_{2} F^{\prime \prime \prime}\left(\mathbf{r}, \mathbf{r}_{1}, \mathbf{r}_{2}\right)
\end{aligned}
$$

and higher-order equations of the total-energy hierarchy; the first,

$$
\begin{aligned}
v_{x c}(\mathbf{r})+T_{c}^{\prime}(\mathbf{r})= & -\mathbf{r} \cdot \boldsymbol{\nabla} v_{x c}(\mathbf{r}) \\
& -\int d \mathbf{r}_{1} n\left(\mathbf{r}_{1}\right) \mathbf{r}_{1} \cdot \nabla_{1} v_{x c}^{\prime}\left(\mathbf{r}, \mathbf{r}_{1}\right),
\end{aligned}
$$

the second,

$$
\begin{aligned}
v_{x c}^{\prime}\left(\mathbf{r}, \mathbf{r}_{1}\right)+T_{c}^{\prime \prime}\left(\mathbf{r}, \mathbf{r}_{1}\right)= & -\left(\mathbf{r} \cdot \boldsymbol{\nabla}+\mathbf{r}_{1} \cdot \nabla_{1}\right) v_{x c}^{\prime}\left(\mathbf{r}, \mathbf{r}_{1}\right) \\
& -\int d \mathbf{r}_{2} n\left(\mathbf{r}_{2}\right) \mathbf{r}_{2} \cdot \nabla_{2} v_{x c}^{\prime \prime}\left(\mathbf{r}, \mathbf{r}_{1}, \mathbf{r}_{2}\right),
\end{aligned}
$$

and higher-order equations of the exchange-correlationenergy hierarchy; and forms of the virial equations,

$$
\begin{aligned}
F-\int n(\mathbf{r}) F^{\prime}(\mathbf{r}) d r+\frac{1}{2} \int n(\mathbf{r}) n\left(\mathbf{r}_{1}\right) F^{\prime \prime}\left(\mathbf{r}, \mathbf{r}_{1}\right) d \mathbf{r} d \mathbf{r}_{1}- & \cdots \\
= & -\left[T-\int n(\mathbf{r}) T^{\prime}(\mathbf{r}) d \mathbf{r}+\frac{1}{2} \int n(\mathbf{r}) n\left(\mathbf{r}_{1}\right) T^{\prime \prime}\left(\mathbf{r}, \mathbf{r}_{1}\right) d \mathbf{r} d \mathbf{r}_{1}-\cdots\right]
\end{aligned}
$$

and

$$
\begin{array}{rl}
E_{x c}-\int n(\mathbf{r}) v_{x c}(\mathbf{r}) d r+\frac{1}{2} \int n(\mathbf{r}) n\left(\mathbf{r}_{1}\right) v_{x c}^{\prime}\left(\mathbf{r}, \mathbf{r}_{1}\right) d \mathbf{r} & d \mathbf{r}_{1}-\cdots \\
& =-\left[T_{c}-\int n(\mathbf{r}) T_{c}^{\prime}(\mathbf{r}) d \mathbf{r}+\frac{1}{2} \int n(\mathbf{r}) n\left(\mathbf{r}_{1}\right) T_{c}^{\prime \prime}\left(\mathbf{r}, \mathbf{r}_{1}\right) d \mathbf{r} d \mathbf{r}_{1}-\cdots\right]
\end{array}
$$

The prime in these equations denotes the functional derivative with respect to the density $n$. $F$ contains the kinetic-energy functional $T$ plus the total electronelectron potential energy $V_{e e} . \quad v_{x c}$ and $E_{x c}$ are the exchange-correlation potential and energy, respectively. $T_{c}=T-T_{s}$ is the difference of the interacting and noninteracting kinetic-energy functionals.

The hierarchy of equations for the noninteracting kinetic and the exchange energies and derived and solved in the local-density approximation. The truncation of these hierarchies provides bounds to the kinetic and exchange energies.

\section{HIERARCHY OF EQUATIONS FOR THE TOTAL ENERGY FUNCTIONAL, THE HARDNESS KERNEL, AND THE LOCAL HARDNESS}

Let us consider a system of $N$ interacting electrons moving in an external field $v(\mathbf{r})$. The ground-state energy is

$$
\begin{aligned}
E & =\int d \mathbf{r} n(\mathbf{r}) v(\mathbf{r})+F[n] \\
& =\int d \mathbf{r} n(\mathbf{r}) v(\mathbf{r})+T[n]+V_{e e},
\end{aligned}
$$


where the kinetic energy $T$ and the electron-electron energy are unique functionals of the electron density $n$. Introducing the quantity $\Omega$ defined as

$$
\Omega=E-\mu N=F[n]+\int d \mathbf{r} n(\mathbf{r})[v(\mathbf{r})-\mu],
$$

the minimization of $\Omega$ leads to the Euler-Lagrange equation

$$
\left.\frac{\delta \Omega}{\delta n}\right|_{v, \mu}=\left.\frac{\delta F}{\delta n}\right|_{v, \mu}+v(\mathbf{r})-\mu=0,
$$

where the Lagrange multiplier $\mu$ is the chemical potential and is determined by the condition that

$$
\int d \mathbf{r} n(\mathbf{r})=N
$$

is the number of electrons. The functional equation (9) can be written as

$$
F^{\prime}(\mathbf{r} ; n)=\mu-v(\mathbf{r})=-u(\mathbf{r})
$$

where

$$
\left.F^{\prime}(\mathbf{r} ; n) \equiv \frac{\delta F[n]}{\delta n(\mathbf{r})}\right|_{v, \mu} .
$$

Taking the gradient of the Euler-Lagrange equation (11) we arrive at

$$
\nabla u(\mathbf{r})=-\nabla F^{\prime}(\mathbf{r} ; n) .
$$

The functional differentiation with respect to $n(\mathbf{r})$ of the universal virial relation of Levy and Perdew [2],

$$
F[n]+T[n]=-\int d \mathbf{r} n(\mathbf{r}) \mathbf{r} \cdot \nabla F^{\prime}(\mathbf{r} ; n),
$$

gives

$$
\begin{aligned}
F^{\prime}(\mathbf{r} ; n)+T^{\prime}(\mathbf{r} ; n)= & -\mathbf{r} \cdot \nabla F^{\prime}(\mathbf{r} ; n) \\
& -\int d \mathbf{r}_{1} n\left(\mathbf{r}_{1}\right) \mathbf{r}_{1} \cdot \nabla_{1} \eta\left(\mathbf{r}, \mathbf{r}_{1} ; n\right) .
\end{aligned}
$$

The hardness kernel [3]

$$
\begin{aligned}
\eta\left(\mathbf{r}, \mathbf{r}_{1} ; n\right)=F^{\prime \prime}\left(\mathbf{r}, \mathbf{r}_{1} ; n\right) & =\frac{\delta F^{\prime}(\mathbf{r} ; n)}{\delta n\left(\mathbf{r}_{1}\right)} \\
& =\frac{\delta^{2} F[n]}{\delta n(\mathbf{r}) \delta n\left(\mathbf{r}_{1}\right)}=-\frac{\delta u(\mathbf{r} ; n)}{\delta n\left(\mathbf{r}_{1}\right)}
\end{aligned}
$$

is the second functional derivate of $F[n]$ which can equivalently be given by

$$
\eta\left(\mathbf{r}, \mathbf{r}_{1} ; n\right)=\frac{\delta^{2} \Omega}{\delta n(\mathbf{r}) \delta n\left(\mathbf{r}_{1}\right)}
$$

$\eta$ is positive definite because $\Omega$ takes a minimum at the density $n(\mathbf{r})$ determined by the Euler-Lagrange equation (9). Functional differentiation with respect to $n$ ( $\mathbf{r}$ ) of Eq. (15) provides

$$
\begin{aligned}
\eta\left(\mathbf{r}, \mathbf{r}_{1} ; n\right)+T^{\prime \prime}\left(\mathbf{r}, \mathbf{r}_{1} ; n\right) \\
=-\left(\mathbf{r} \cdot \boldsymbol{\nabla}+\mathbf{r}_{1} \cdot \nabla_{1}\right) \eta\left(\mathbf{r}, \mathbf{r}_{1} ; n\right) \\
\quad-\int d \mathbf{r}_{2} n\left(\mathbf{r}_{2}\right) \mathbf{r}_{2} \cdot \nabla_{2} \eta^{\prime}\left(\mathbf{r}, \mathbf{r}_{1}, \mathbf{r}_{2} ; n\right),
\end{aligned}
$$

where

$$
\begin{aligned}
\eta^{\prime}\left(\mathbf{r}, \mathbf{r}_{1}, \mathbf{r}_{2} ; n\right)=\frac{\delta n\left(\mathbf{r}, \mathbf{r}_{1} ; n\right)}{\delta n\left(\mathbf{r}_{2}\right)} & =F^{\prime \prime \prime}\left(\mathbf{r}, \mathbf{r}_{1}, \mathbf{r}_{2} ; n\right) \\
& =\frac{\delta^{3} F[n]}{\delta n(\mathbf{r}) \delta n\left(\mathbf{r}_{1}\right) \delta n\left(\mathbf{r}_{2}\right)}
\end{aligned}
$$

Equations (14), (15), and (18) constitute the zeroth, first, and second equations of the hierarchy, respectively. Further differentiations will lead to higher-order equations.

The virial expression (14) can be rewritten with the aid of the hierarchy $(15)-(18)$,

$$
\begin{aligned}
F[n]-\int n(\mathbf{r}) F^{\prime}(\mathbf{r} ; n) d \mathbf{r}+d r \frac{1}{2} \int n(\mathbf{r}) n\left(\mathbf{r}_{1}\right) & \eta\left(\mathbf{r}, \mathbf{r}_{1} ; n\right) d \mathbf{r} d \mathbf{r}_{1}-\ldots \\
& =-\left[T[n]-\int n(\mathbf{r}) T^{\prime}(\mathbf{r} ; n) d r+\frac{1}{2} \int n(\mathbf{r}) n\left(\mathbf{r}_{1}\right) T^{\prime \prime}\left(\mathbf{r}, \mathbf{r}_{1} ; n\right) d \mathbf{r} d \mathbf{r}_{1} \cdots\right],
\end{aligned}
$$

as an alternating series. Introducing the local hardness [4] $\eta(\mathbf{r})$ as

$$
\eta(\mathbf{r})=\frac{1}{N} \int \eta\left(\mathbf{r}, \mathbf{r}_{1} ; n\right) n\left(\mathbf{r}_{1}\right) d \mathbf{r}_{1}
$$

Eq. (20) takes the form

$$
\begin{aligned}
F[n]-\int n(\mathbf{r}) F^{\prime}(\mathbf{r} ; n) d \mathbf{r}+\frac{N}{2} \int n(\mathbf{r}) \eta(\mathbf{r}) & d \mathbf{r}-\cdots \\
= & -\left[T[n]-\int n(\mathbf{r}) T^{\prime}(\mathbf{r} ; n) d r+\frac{1}{2} \int n(\mathbf{r}) n\left(\mathbf{r}_{1}\right) T^{\prime \prime}\left(\mathbf{r}, \mathbf{r}_{1} ; n\right) d \mathbf{r} d \mathbf{r}^{\prime}-\cdots\right] .
\end{aligned}
$$

Using the definition of the local hardness [Eq. (21)], Eq. (15) can be also written as

$$
F^{\prime}(\mathbf{r} ; n)+T^{\prime}(\mathbf{r} ; n)=-\mathbf{r} \cdot \nabla F^{\prime}(\mathbf{r} ; n)+3 N \eta(\mathbf{r})+\int d \mathbf{r}_{1} \eta\left(\mathbf{r}, \mathbf{r}_{1} ; n\right) \mathbf{r}_{1} \cdot \nabla_{1} n\left(\mathbf{r}_{1}\right) .
$$

This last equation is especially interesting as it contains explicitly the local hardness $\eta(\mathbf{r})$. 


\section{HIERARCHY OF EQUATIONS FOR THE NONINTERACTING KINETIC- ENERGY FUNCTIONAL}

Kugler [1] derived hierarchy of equations for the kinetic energy of noninteracting electrons. Now, we review the most important results relevant here. The starting point is the virial relation

$$
T_{s}[n]=\frac{1}{2} \int d \mathbf{r} n(\mathbf{r}) \mathbf{r} \cdot \nabla v_{\mathrm{KS}},
$$

where the potential $v_{\mathrm{KS}}$ denotes the common Kohn-Sham potential field in which the noninteracting electrons move. The Euler-Lagrange equation (9) can be rewritten as

$$
\frac{\delta T_{s}[n]}{\delta n(\mathbf{r})}+v_{\mathrm{KS}}-\mu=0,
$$

if the functional $F[n]$ is partitioned into

$$
F[n]=T_{s}[n]+U_{c}[n]+E_{x c}[n],
$$

where the electron-electron Coulomb energy has the usual form

$$
U_{c}[n]=\frac{1}{2} \int \frac{n(\mathbf{r}) n\left(\mathbf{r}_{1}\right)}{\left|\mathbf{r}-\mathbf{r}_{1}\right|} d \mathbf{r} d \mathbf{r}_{1},
$$

while the exchange-correlation energy contains a kineticenergy contribution,

$$
T_{c}[n]=T[n]-T_{s}[n],
$$

corresponding to the difference in the kinetic energies of the interacting and noninteracting electrons. Combining Eqs. (24) and (25) we arrive at the zeroth equation of the hierarchy of the kinetic energy,

$$
T_{s}[n]=-\frac{1}{2} \int d \mathbf{r} n(\mathbf{r}) \mathbf{r} \cdot \nabla T_{s}^{\prime}(\mathbf{r} ; n)
$$

Functional differentiation with respect to $n(\mathbf{r})$ of Eq. (29) leads to

$$
\begin{aligned}
T_{s}^{\prime}(\mathbf{r} ; n)= & -\frac{1}{2} \mathbf{r} \cdot \nabla T_{s}^{\prime}(\mathbf{r} ; n) \\
& -\frac{1}{2} \int d \mathbf{r}_{1} n\left(\mathbf{r}_{1}\right) \mathbf{r}_{1} \cdot \nabla_{1} T_{s}^{\prime \prime}\left(\mathbf{r}, \mathbf{r}_{1} ; n\right)
\end{aligned}
$$

Equation (30) has also been derived recently by Ou-Yang and Levy [8]. Again, functionally differentiating Eq. (30) we obtain

$$
\begin{aligned}
T_{s}^{\prime \prime}\left(\mathbf{r}, \mathbf{r}_{1} ; n\right)= & -\frac{1}{2}\left(\mathbf{r} \cdot \boldsymbol{\nabla}+\mathbf{r}_{1} \cdot \nabla_{1}\right) T_{s}^{\prime \prime}\left(\mathbf{r}, \mathbf{r}_{1} ; n\right) \\
& -\frac{1}{2} \int d \mathbf{r}_{2} n\left(\mathbf{r}_{2}\right) \mathbf{r}_{2} \cdot \nabla_{2} T_{s}^{\prime \prime \prime}\left(\mathbf{r}, \mathbf{r}_{1}, \mathbf{r}_{2} ; n\right) .
\end{aligned}
$$

Equations (29), (30), and (31) constitute the zeroth, first, and second equations of the hierarchy of the noninteracting kinetic energy. Obviously, higher-order equations can similarly be derived, too.

Following Kugler [1] we can rewrite the virial theorem (24) with the aid of the hierarchy (30) and (31) as

$$
\begin{array}{rl}
T_{s}[n]=\int d \mathbf{r} & n(\mathbf{r}) T_{s}^{\prime}(\mathbf{r} ; n) \\
& -\frac{1}{2} \int d \mathbf{r} d \mathbf{r}_{1} n(\mathbf{r}) n\left(\mathbf{r}_{1}\right) T_{s}^{\prime \prime}\left(\mathbf{r}, \mathbf{r}_{1} ; n\right)+\cdots .
\end{array}
$$

The alternating series (32) contains only the variational derivatives of the noninteracting kinetic energy.

\section{HIERARCHY OF EQUATIONS FOR THE EXCHANGE-CORRELATION ENERGY FUNCTIONAL}

Combining the virial relations (14) and (24) the LevyPerdew relation for the exchange-correlation energy can be obtained,

$$
E_{x c}[n]+T_{c}[n]=-\int d \mathbf{r} n(\mathbf{r}) \mathbf{r} \cdot \nabla v_{x c}(\mathbf{r} ; n),
$$

where

$$
v_{x c}(\mathbf{r} ; n)=\frac{\delta E_{x c}[n]}{\delta n(\mathbf{r})}
$$

is the exchange-correlation potential. Again, by functional differentiation, we get the first equation of the hierarchy of the exchange-correlation energy,

$$
\begin{aligned}
v_{x c}(\mathbf{r} ; n)+T_{c}^{\prime}(\mathbf{r} ; n)= & -\mathbf{r} \cdot \nabla v_{x c}(\mathbf{r} ; n) \\
& -\int d \mathbf{r}_{1} n(\mathbf{r}) \mathbf{r}_{1} \cdot \nabla_{1} v_{x c}^{\prime}\left(\mathbf{r}, \mathbf{r}_{1} ; n\right) .
\end{aligned}
$$

The second equation of the hierarchy can be given by

$$
\begin{aligned}
& v_{x c}^{\prime}\left(\mathbf{r}, \mathbf{r}_{1} ; n\right)+T_{c}^{\prime \prime}\left(\mathbf{r}, \mathbf{r}_{1} ; n\right) \\
&=-\left(\mathbf{r} \cdot \boldsymbol{\nabla}+\mathbf{r}_{1} \cdot \nabla_{1}\right) v_{x c}^{\prime}\left(\mathbf{r}, \mathbf{r}_{1} ; n\right) \\
& \quad-\int d \mathbf{r}_{2} n\left(\mathbf{r}_{2}\right) \mathbf{r}_{2} \cdot \nabla_{2} v_{x c}^{\prime \prime}\left(\mathbf{r}, \mathbf{r}_{1}, \mathbf{r}_{2} ; n\right) .
\end{aligned}
$$

Further functional differentiation leads to successively higher-order equations. The Levy-Perdew relation [2] (33) can be rewritten with the aid of Eqs. (35) and (36),

$$
\begin{aligned}
E_{x c}[n]-\int n(\mathbf{r}) v_{x c}(\mathbf{r} ; n) d \mathbf{r}+\frac{1}{2} \int n(\mathbf{r}) n\left(\mathbf{r}_{1}\right) v_{x c}^{\prime}\left(\mathbf{r}, \mathbf{r}_{1} ; n\right) d \mathbf{r} d \mathbf{r}_{1}-\cdots \\
=-\left[T_{c}[n]-\int n(\mathbf{r}) T_{c}^{\prime}(\mathbf{r} ; n) d \mathbf{r}+\frac{1}{2} \int n(\mathbf{r}) n\left(\mathbf{r}_{1}\right) T_{c}^{\prime \prime}\left(\mathbf{r}, \mathbf{r}_{1} ; n\right) \mathbf{r} d \mathbf{r}^{\prime}-\cdots\right]
\end{aligned}
$$

\section{HIERARCHY OF EQUATIONS FOR THE EXCHANGE-ENERGY FUNCTIONAL}

Unfortunately, the hierarchy of equations for the exchange-correlation energy contains the unknown kinetic-energy contribution $T_{c}$. As $T_{c} \approx-E_{c}$, where $E_{c}$ is the correlation energy, it cannot generally be neglected. However, in an exchange-only approximation it is enough using the hierarchy of equations for the exchange. Starting out from the Levy-Perdew relation [2], 


$$
E_{x}[n]=-\int d \mathbf{r} n(\mathbf{r}) \mathbf{r} \cdot \nabla v_{x}(\mathbf{r} ; n)
$$

which is the zeroth equation of the hierarchy, we get the first equation,

$$
v_{x}(\mathbf{r} ; n)=-\mathbf{r} \cdot \nabla v_{x}(\mathbf{r} ; n)-\int d \mathbf{r}_{1} n\left(\mathbf{r}_{1}\right) \mathbf{r}_{1} \cdot \nabla_{1} v_{x}^{\prime}\left(\mathbf{r}, \mathbf{r}_{1} ; n\right),
$$

and the second equation,

$$
\begin{aligned}
v_{x}^{\prime}\left(\mathbf{r}, \mathbf{r}_{1} ; n\right)= & -\left(\mathbf{r} \cdot \boldsymbol{\nabla}+\mathbf{r}_{1} \cdot \nabla_{1}\right) v_{x}^{\prime}\left(\mathbf{r}, \mathbf{r}_{1} ; n\right) \\
& -\int d \mathbf{r}_{2} n\left(\mathbf{r}_{2}\right) \mathbf{r}_{2} \cdot \nabla_{2} v_{x}^{\prime \prime}\left(\mathbf{r}, \mathbf{r}_{1}, \mathbf{r}_{2} ; n\right)
\end{aligned}
$$

Expression (39) has been recently obtained by Ou-Yang and Levy [8]. Using Eqs. (39) and (40) the Levy-Perdew relation [2] (38) can be also been written as

$$
\begin{aligned}
E_{x}[n]= & \int n(\mathbf{r}) v_{x}(\mathbf{r} ; n) d \mathbf{r} \\
& -\frac{1}{2} \int n(\mathbf{r}) n\left(\mathbf{r}_{1}\right) v_{x}^{\prime}\left(\mathbf{r}, \mathbf{r}_{1} ; n\right) d \mathbf{r} d \mathbf{r}_{1}+\cdots .
\end{aligned}
$$

\section{APPLICATION: THE LOCAL-DENSITY APPROXIMATION}

As an application the local-density approximation is studied. In this approximation the kinetic-energy density

$$
t(\mathbf{r})=t(n(\mathbf{r}))
$$

and the exchange or exchange-correlation energy

$$
\begin{aligned}
& e_{x}(\mathbf{r})=e_{x}(n(\mathbf{r})), \\
& e_{x c}(\mathbf{r})=e_{x c}(n(\mathbf{r}))
\end{aligned}
$$

are functions of the density $n$. The first and second functional derivatives of the kinetic energy are

$$
T_{s}^{\prime}=\frac{d t}{d n}
$$

and

$$
T_{s}^{\prime \prime}\left(\mathbf{r}, \mathbf{r}_{1}\right)=\frac{d^{2} t}{d n^{2}} \delta\left(\mathbf{r}, \mathbf{r}_{1}\right),
$$

respectively. Substituting Eqs. (45) and (46) into the first equation of the hierarchy of the noninteracting kinetic energy (30) we arrive at

$$
\frac{d t}{d n}-=-\frac{1}{2} \mathbf{r} \cdot \nabla \frac{d t}{d n}+\frac{1}{2} \frac{d^{2} t}{d n^{2}} \nabla(\mathbf{r} n)
$$

Applying Eq. (42),

$$
\nabla \frac{d t}{d n}=\frac{d^{2} t}{d n^{2}} \nabla n
$$

which leads to the differential equation for the kineticenergy density,

$$
\frac{d t}{d n}=\frac{3}{2} n \frac{d^{2} t}{d n^{2}} .
$$

The solution is

$$
t=c n^{5 / 3} \text {, }
$$

i.e., the well-known Thomas-Fermi expression.

The exchange potential and its functional derivate are

$$
v_{x}=\frac{d e_{x}}{d n}
$$

and

$$
v_{x}^{\prime}\left(\mathbf{r}, \mathbf{r}_{1}\right)=\frac{d^{2} e_{x}}{d n^{2}} \delta\left(\mathbf{r}, \mathbf{r}_{1}\right),
$$

respectively. The substitution of Eqs. (51) and (52) into the first equation of the hierarchy of the exchange energy (39) leads to the equation

$$
\frac{d e_{x}}{d n}=-\mathbf{r} \cdot \nabla \frac{d e_{x}}{d n}+\frac{d^{2} e_{x}}{d n^{2}} \nabla(n \mathbf{r}) .
$$

With the identity

$$
\nabla \frac{d e_{x}}{d n}=\frac{d^{2} e_{x}}{d n^{2}} \nabla n,
$$

Eq. (53) gives

$$
\frac{d e_{x}}{d n}=3 n \frac{d^{2} e_{x}}{d n^{2}} .
$$

The solution of this differential equation,

$$
e_{x}=a n^{4 / 3}
$$

and

$$
v_{x}=\frac{4}{3} a n^{1 / 3},
$$

gives the exchange potential proposed by Slater [5], Gáspár [6], and Kohn and Sham [7]. So, in the localdensity approximation the solutions of the equations of hierarchy for the kinetic and exchange energies provide the well-known Thomas-Fermi expression for the kinetic energy and the Slater-Gáspár-Kohn-Sham expression for the exchange.

\section{TRUNCATION OF THE HIERARCHY OF THE EQUATIONS FOR THE KINETIC- AND EXCHANGE-ENERGY FUNCTIONALS}

It is possible to introduce approximations truncating the hierarchy of equations for the energy functionals at a given order. Considering first the exchange and truncating the hierarchy with assuming

$$
v_{x}^{\prime}\left(\mathbf{r}, \mathrm{r}_{1} ; n\right) \approx 0,
$$

Eq. (39) has the form

$$
v_{x}=-\mathbf{r} \cdot \boldsymbol{\nabla} v_{x}
$$

Using the identity [8]

$$
\mathbf{r} \cdot \boldsymbol{\nabla}=r \frac{\partial}{\partial r},
$$

the first equation of the hierarchy is given by

$$
v_{x}=-r \frac{\partial v_{x}}{\partial r} \text {. }
$$


The solution of Eq. (61) is

$$
v_{x}=\frac{B}{r}
$$

and it leads to the exchange energy

$$
E_{x}=B \int n \frac{1}{r} d \mathbf{r},
$$

where $B$ is constant. This result can be generalized. Truncating the hierarchy at the $k$ th order, i.e., neglecting the $(k+1)$ th functional derivate of the exchange energy, the exchange energy has the form

$$
E_{x}=B_{k}\left\langle r^{-(1 / k)}\right\rangle^{k},
$$

where

$$
\left\langle r^{-(1 / k)}\right\rangle=\int r^{-(1 / k)} n(\mathbf{r}) d \mathbf{r}
$$

and $B_{k}$ is constant.

A similar procedure can be applied to the kineticenergy hierarchy. Truncating the hierarchy at the $k$ th order, after some algebra, one can arrive at the kineticenergy expression

$$
T_{s}=C_{k}\left\langle r^{-(2 / k)}\right\rangle^{k},
$$

where $C_{k}$ is constant.

Recently Dehesa, Galvez, and Porras [9] derived rigorous bounds to the kinetic and exchange energies. They found several expressions for lower bounds to the kinetic and upper bounds to the exchange energies in the plane-wave approximation. Their Eqs. (12a) and (13a) with appropriate choice of the parameters lead to the inequalities

$$
T_{s} \geq \widetilde{C}_{k}\left\langle r^{-(2 / k)}\right\rangle^{k}
$$

and

$$
K_{x} \leq \widetilde{B}_{k}\left\langle r^{-(1 / k)}\right\rangle^{k},
$$

where $\widetilde{C}_{k}$ and $\widetilde{B}_{k}$ depend only on the number of electrons, i.e., are constant for a given system. Choosing $B_{k}=\widetilde{B}_{k}$ and $C_{k}=\widetilde{C}_{k}$ we arrive at the very interesting results that the truncation of the hierarchies of the kinetic and exchange energies leads to expressions that can be used to rigorously bound the kinetic and exchange energies in the plane-wave approximations.

\section{DISCUSSION}

The hierarchies of equations derived for the energy functionals can be used to obtain more accurate function- al expressions. The equations presented here may prove useful comparing and testing various forms of approximating functionals.

The hierarchies of equations obtained resemble the Bogoliubov-Born-Green-Kirkwood-Yvon hierarchy. In Sec. VII the possibility of truncation is studied in the kinetic- and exchange-energy hierarchies. The truncation leads to lower bounds to the kinetic and upper bounds to the exchange energies.

Equation (23) contains the local hardness and the hardness kernel. The hardness has proved to be an important concept in chemistry. It is difficult to calculate the hardness and the hardness kernel as the exact form of the functional $F$ is not known. Equation (23) may be a useful starting point for some new approximations for the local hardness or the hardness kernel.

From Eqs. (16), (26), and (27) we get for the hardness kernel

$\eta\left(\mathbf{r}, \mathbf{r}_{1} ; n\right)=T_{s}^{\prime \prime}\left(\mathbf{r}, \mathbf{r}_{1} ; n\right)+v_{x c}^{\prime}\left(\mathbf{r}, \mathbf{r}_{1} ; n\right)+U_{c}^{\prime \prime}\left(\mathbf{r}, \mathbf{r}_{1} ; n\right)$,

where

$$
U_{c}^{\prime \prime}\left(\mathbf{r}, \mathbf{r}_{1} ; n\right)=\frac{\delta^{2} U_{c}[n]}{\delta n(\mathbf{r}) \delta n\left(\mathbf{r}_{1}\right)}=\frac{1}{\left|\mathbf{r}-\mathbf{r}_{1}\right|} .
$$

As it can be seen from the derivation and the results above the hierarchy of equations links the $n$th functional derivatives to the $(n+1)$ th functional derivate and the electron density. In the case of the noninteracting kinetic-energy (or the exchange-energy) functional, the hierarchy of equations are "self-contained" in the sense that they contain only the kinetic- (or the exchange-) energy functional and its functional derivatives. It is the consequence of the fact that both the noninteracting kinetic energy and the exchange-energy functional scale homogeneously as it has been shown by Ou-Yang and Levy [8].

On the other hand, the equations of hierarchy for the total energy and the exchange-correlation energy functionals are much more complicated because they are coupled in the sense that they contain more than one functional and its functional derivatives.

\section{ACKNOWLEDGMENTS}

The author is grateful to Professor Robert G. Parr for warm hospitality, many valuable discussions, encouragement and suggestions. This publication is based on work sponsored by the Hungarian-U.S. Science and Technology Joint Fund in cooperation with National Science Foundation and Hungarian Academy of Sciences under Project 146/91.
[1] A. A. Kugler, Phys. Rev. A 41, 3489 (1990).

[2] M. Levy and J. P. Perdew, Phys. Rev. A 32, 2010 (1985).

[3] M. Berkowitz and R. G. Parr, J. Chem. Phys. 88, 2553 (1988).

[4] S. K. Ghosh and M. Berkowitz, J. Chem. Phys. 83, 2976 (1985).
[5] J. C. Slater, Phys. Rev. 81, 385 (1951).

[6] R. Gáspár, Acta Phys. Acad. Sci. Hung. 3, 263 (1954).

[7] W. Kohn and L. J. Sham, Phys. Rev. A 140, 1133 (1965).

[8] H. Ou-Yang and M. Levy, Phys. Rev. A 44, 54 (1991).

[9] J. S. Dehesa, F. J. Galvez, and I. Porras, Phys. Rev. A 40, 35 (1989). 